\title{
Evolución de la investigación en el campo de las ciencias militares aeronáuticas en Colombia ${ }^{1}$
}

\author{
https://doi.org/10.21830/9789585380202.01
}

\author{
Diana Paola Brochero Barragán ${ }^{2}$ \\ Nora Patricia Gutiérrez Rodriguez ${ }^{3}$ \\ Erika Juliana Estrada Villa ${ }^{4}$
}

Escuela de Postgrados Fuerza Aérea Colombiana

\begin{abstract}
Resumen. En este capítulo se describe la trayectoria investigativa en ciencias militares aeronáuticas de la Maestría en Ciencias Militares Aeronáuticas (MACMA) de la Escuela de Postgrados de la Fuerza Aérea Colombiana a partir de los trabajos de grado de sus estudiantes. La investigación tuvo un enfoque cualitativo, mediante el cual se analizaron los programas académicos similares a la MACMA desde dos puntos de vista: las actividades que desarrollan en el perfil profesional relacionado con la administración de organizaciones y recursos militares, y la reglamentación de la Ley de Educación Superior, Ciencia y Tecnología, así como las normativas y reglamentos del Ministerio de Educación. De acuerdo con los trabajos de grado elaborados en la МАCMA, se concluye que la trayectoria investigativa de las ciencias militares aeronáuticas ha tendido al estudio de los siguientes temas: UAV-drones, manuales y técnicas, proyección, capacidades y capacidades distintivas.
\end{abstract}

Palabras clave: ciencias militares aeronáuticas; doctrina; enseñanza superior; investigación; normatividad; programas académicos.

1 Este capítulo forma parte de los resultados del proyecto de investigación "Consideraciones sobre la investigación en el campo de las ciencias militares aeronáuticas", del Grupo de Investigación en Ciencias Militares Aeronáuticas y Administrativas (Gicma) de la Escuela de Postgrados de la Fuerza Aérea Colombiana, Colombia, categorizado en C por Minciencias y con código de registro COL0140489. Los puntos de vista que se presentan en este capítulo pertenecen a los autores y no reflejan necesariamente los de las instituciones participantes.

2 Mayor de la Fuerza Aérea Colombiana. Estudiante de la Maestría en Ciencias Militares Aeronáuticas de la Escuela de Postgrados de la Fuerza Aérea Colombiana. Odontóloga y especialista en Patología y Cirugía Bucal de la Pontificia Universidad Javeriana. OrCiD: https://orcid.org/0000-0002-12026895 - Contacto: diana.brochero@epfac.edu.co

3 Mayor de la Fuerza Aérea Colombiana. Magíster en Historia de la Universidad del Valle. Directora de la Maestría en Ciencias Militares Aeronáuticas de la Escuela de Postgrados de la Fuerza Aérea Colombiana. OrCiD: https://orcid.org/0000-0002-6795-5093 - Contacto: nora.gutierrez@epfac.edu.co

4 Magíster en Informática Educativa de la Universidad de La Sabana. Docente de Investigación de la Escuela de Postgrados Fuerza Aérea Colombiana. Orcid: https://orcid.org/0000-0001-5445-2895 Contacto: erika.estrada@epfac.edu.co 


\section{Introducción}

A grandes rasgos, el concepto de ciencias militares agrupa disciplinas de orden científico, tecnológico, estratégico y social que se interrelacionan con el fin de mantener el orden, la seguridad y la paz.

Un territorio con un historial de conflictos y problemáticas como el nuestro necesita profesionales altamente preparados para enfrentar esos desafíos, esto es, personas capaces de encontrar los medios tecnológicos, humanísticos y científicos para alcanzar los ideales de paz y orden con sentido ético y social. En suma, sujetos con espíritu y capacidad investigativa, curiosos no solo por lo que sucede en el ámbito social y militar donde desempeñan sus tareas, sino también por lo que traen los continuos desarrollos de las ciencias. Por ello, este documento se orienta a determinar cuál es la trayectoria investigativa de las ciencias militares aeronáuticas (CMA) en Colombia para la toma de decisiones, en los programas académicos de nivel posgradual de la Fuerza Aérea Colombiana (FAC).

La competencia investigativa, es decir, la capacidad del profesional para producir conocimiento siguiendo los pasos del método científico con el fin de encontrar soluciones a problemas del entorno, de acuerdo con Reiban et al. (2017), puede tener diferentes enfoques y perspectivas: el nivel de los sujetos de estudio (por ejemplo, estudiantes, profesionales, especialistas o doctores), una problemática en particular o el empleo de herramientas para la adquisición de las competencias.

La competencia entonces emerge de la intersección entre los conocimientos factuales y declarativos (saber conocer), habilidades y destrezas (saber hacer), y actitudes y valores (saber ser), y sustenta su existencia en la necesidad de resolver problemas y situaciones (Pimienta, 2012). En este sentido, Tobón (2008) propone que una política clave para la educación superior es la formación por competencias, y entiende las competencias como "procesos complejos de desempeño ante problemas con idoneidad y compromiso ético" (p. 1), que le permiten al profesional resolver de forma acertada los desafíos de su labor. Esto exige planes generales e integrativos de aprendizaje y evaluación, orientados a que los estudiantes desarrollen varias competencias establecidas en el perfil académico profesional de egreso de un determinado programa. Por su 
parte, Morin (2001) menciona que las competencias abordan el desempeño de manera compleja, teniendo como referencia la realización de actividades y la resolución de problemas de diferentes contextos (disciplinares, sociales, científicos y profesionales-laborales). A su vez, Pimienta (2012) plantea que las competencias existen por la necesidad de resolver problemas y situaciones. Y Pérez (2012) percibe las competencias del ser, saber y saber hacer como procesos de enseñanza-aprendizaje que permiten estructurar currículos para la vida, desarrollar potencialidades, satisfacer las necesidades del ser humano y mejorar la capacidad de gestión con una mayor calidad organizativa y administrativa.

Asimismo, la "doctrina militar" aplicada al ámbito de la educación castrense y policial se entiende como "el vector principal que orienta el desarrollo de los contenidos de los programas de estudio; además, ella condiciona la definición de los perfiles de los militares y policías, y por consiguiente los contenidos de las mallas curriculares" (Ministerio de Defensa, 2008, p. 25).

Según Ortega (2016),

se entiende entonces que el objeto de estudio de las ciencias militares implica la observación y sistematización de las informaciones relacionadas con los fenómenos que tratan sobre la guerra y el empleo de los medios militares en el proceso de gestación, desarrollo y evolución de un conflicto, incluyendo las actividades relacionadas con la prevención de la guerra o articulación de la paz. (p. 26)

El análisis sistemático y permanente de estos fenómenos, guerras, conflictos, etc., produce paradigmas que son utilizados en el campo profesional de los militares y son reconocidos como el conjunto de teorías que orientan el empleo y la conducción de operaciones militares en un contexto regido por las dinámicas de los conflictos armados, incluyendo su prevención.

De esta manera, la doctrina militar se vincula con las dinámicas sociales, y se articula con las necesidades políticas del Estado y con los intereses nacionales de un país. El cambio en estas necesidades o intereses nacionales influye en la transformación, actualización o generación de la doctrina, es decir, produce nuevo conocimiento. Por ello, la doctrina es un aspecto relevante en el campo de las ciencias militares, pues es considerada el fundamento mismo de la filosofía castrense. 
Específicamente, en la FAC la doctrina se entiende como "una afirmación de creencias oficialmente sancionadas, principios de la guerra y terminología que describen y guían el uso del PAEC (poder aéreo y espacial colombiano)" (Fuerza Aérea de Colombia [FAC], 2020, p. 55).

Estas creencias se encuentran en documentos normativos que regulan los procesos y la conducta del profesional militar dentro y fuera del campo de batalla. Por tal razón, esta investigación tiene un enfoque cualitativo-descriptivo, porque se busca interpretar el contexto en el cual surgen las dinámicas y se consolidan las tendencias investigativas en las CMA (Hernández-Sampieri et al., 2010).

Según estos parámetros investigativos, el campo de las CMA en Colombia es liderado por la FAC a través de sus escuelas de formación (pregrado) y capacitación (posgrados), con un enfoque teórico según el cual las CMA son una ciencia multidisciplinaria y transdisciplinaria, que integra diferentes saberes transversales al campo de conocimiento militar y aeronáutico.

Esta diversidad se refleja en la Maestría en Ciencias Militares Aeronáuticas (Macma) de la Escuela de Postgrados de la Fuerza Aérea Colombiana (Epfac), cuya oferta académica contempla en su fundamentación teórica los estudios militares (doctrina militar) y la proyección del poder aéreo a través del desarrollo de diversas investigaciones de tipo formativo que realizan estudiantes de diferentes especialidades y perfiles profesionales, como oficiales del cuerpo de vuelo (pilotos, navegantes), del cuerpo administrativo (trabajadores sociales, economistas, médicos, ingenieros, administradores, etc.) y de otras especialidades militares (inteligencia, logística, seguridad y defensa de bases aéreas).

\section{Marco teórico}

La revisión y el análisis sistemático de las opciones de grado de los estudiantes reflejan igualmente esta correspondencia, que cierra brechas entre saberes, perfiles y productos académicos (producción de conocimiento), al articular y fortalecer la coherencia entre el currículo y las competencias de los profesionales de la maestría, como se verá más adelante.

\section{Educación superior en Colombia}

La Ley 30 de 1992 define la educación superior como "un proceso permanente que posibilita el desarrollo de las potencialidades del ser humano 
de manera integral, [el cual] se realiza con posterioridad a la educación media o secundaria" y su objetivo es "el pleno desarrollo de los estudiantes y su formación académica o profesional" (art. 1) en instituciones de educación superior (cap. IV, art. 16).

Por tanto, los programas de pregrado y de posgrado que ofrezcan las instituciones de educación superior tienen el deber de actuar en los campos de acción que la ley señala y de conformidad con sus propósitos de formación (art. 8). Según esta misma ley, "los programas de pregrado preparan para el desempeño de ocupaciones, para el ejercicio de una profesión o disciplina determinada” (art. 9). A su vez, los programas de maestría tienen como propósito ampliar y desarrollar los conocimientos, actitudes y habilidades para la solución de problemas disciplinares, interdisciplinarios o profesionales, o para dotar a la persona de los instrumentos básicos que la habilitan como investigadora. Según la normativa vigente, los programas podrán ser de profundización o de investigación (Decreto 1330 de 2019).

Para efectos de este capítulo, se aborda una maestría de profundización, de acuerdo con los lineamientos de la sección 6, artículo 2.5.3.2.6.4., del Decreto 1330 de 2019. Este decreto establece que, para optar al título de un programa de maestría en profundización, el estudiante debe cumplir con los requisitos que la institución haya establecido como opción de grado mediante un trabajo de investigación, que podrá ser en forma de estudio de caso, orientado a dar solución a un problema del área funcional (FAC, 2018).

Aunque las ciencias militares no están clasificadas como una disciplina del conocimiento ni se encuentran en la Clasificación Internacional Normalizada de la Educación (Unesco 1996/2006), ni en el Manual de Frascati (OcDe, 2015), Suárez (2005) las define, en un esfuerzo por categorizarlas, como una disciplina contemporánea que surge en la posguerra asociada a las ciencias de la gestión, un conjunto interrelacionado de áreas cuyos métodos y técnicas se emplean para solucionar problemas de la realidad castrense, y que abarca la administración militar, la investigación operativa, la teoría de sistemas, la teoría de la decisión y la planeación estratégica, entre otras.

A su vez, Esquivel (2015) aborda las ciencias militares desde un punto de vista ontológico y realiza un análisis citando a Kuhn (2004) quien menciona que desde lo teórico sigue la propuesta donde la ciencia se rige por sus propias leyes y su reconocimiento subyace a la comunidad que las practica; y fina- 
liza haciendo una reflexión sobre la necesidad de reconstruir cuáles fueron los fundamentos de las ciencias militares que desarrollaron los españoles y que tuvieron adaptaciones en lo geográfico y cultural, por lo que incidieron en la evolución de ese saber.

\section{La Maestría en Ciencias Militares Aeronáuticas. Aproximación y origen}

El programa de la Maestría en Ciencias Militares Aeronáuticas (MACMA) fundamenta su naturaleza académica en la estrategia institucional de consolidar la identidad educativa y garantizar "la formación, actualización, capacitación, instrucción y entrenamiento más adecuados, incorporando una visión estratégica para la educación militar y policial, de tal forma que esta se convierta en el eje estructurador de la cultura institucional" (Ministerio de Defensa, 2008, p. 11).

En atención a lo anterior, mediante la Resolución n. 12382 de 2013, el Ministerio de Educación Nacional aprobó la MACMA, que actualmente tiene una duración de cuatro semestres, lapso en el cual desarrolla el plan de estudios (figura 1).

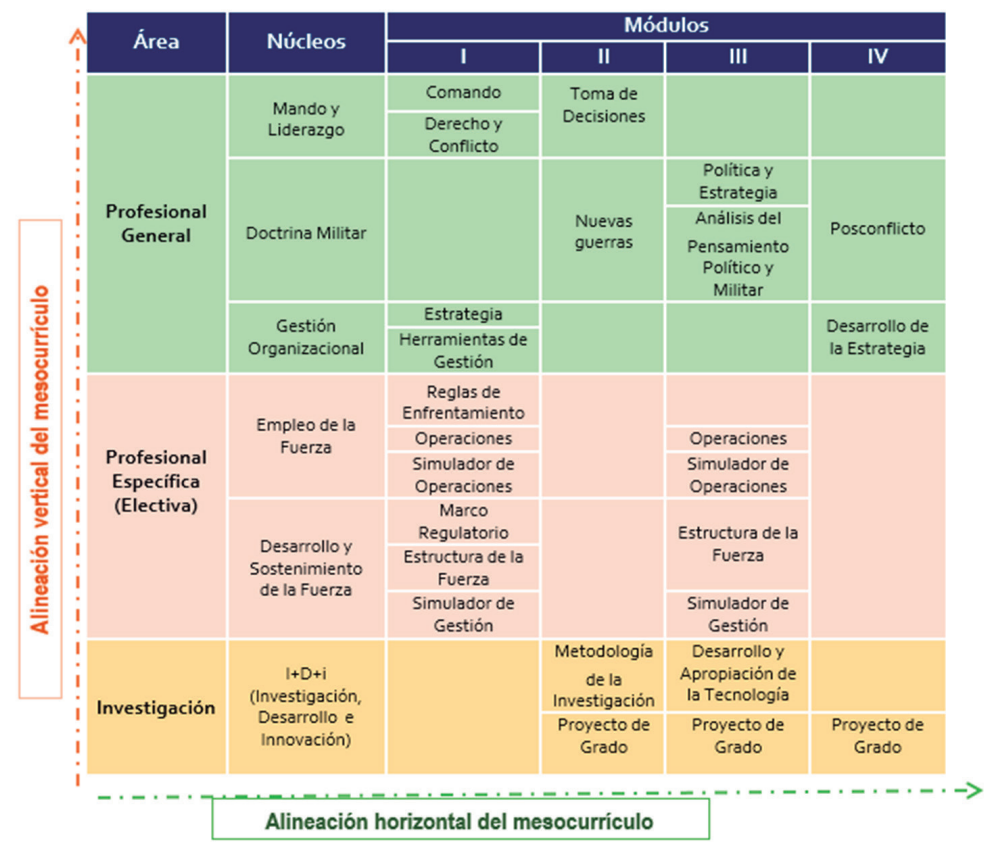

Figura 1. Plan de estudios MACMA para el desarrollo de competencias investigativas. Fuente: Epfac (2019). 
La oferta académica de la MACMa está diseñada teniendo en cuenta los conceptos prácticos y teóricos que contienen las CMA, como liderazgo, estrategia, doctrina, gestión organizacional y conducción de operaciones aéreas (doctrina).

En cuanto a la doctrina militar, este componente del plan de estudios contiene los principios, los criterios y las teorías que orientan el empleo del poder militar. No obstante, ese conocimiento no solo abarca teorías tradicionales, sino también perspectivas de análisis sobre amenazas futuras (nuevas guerras), empleo de los recursos militares (política y estrategia) y de las capacidades del poder aéreo (operaciones y simulador de operaciones).

\section{Método}

De acuerdo con su alcance, la investigación fue descriptiva, dado que realizó un análisis pormenorizado de la realidad, pues "busca especificar las propiedades, las características y los perfiles de personas, grupos, comunidades, procesos, objetos o cualquier otro fenómeno que se someta a un análisis" (Hernández-Sampieri et al., 2010, p. 80). Con el uso de diferentes técnicas y fuentes de información, se buscó caracterizar la trayectoria investigativa en el campo de conocimiento de las CMA a través de la MACMa y como resultado se obtuvieron datos que describen los núcleos temáticos de la maestría.

La población objeto del estudio fue los trabajos de grado de los estudiantes del programa Macma. Se hizo un muestreo por juicio, que, según Mejía (2000), "consiste en la selección de las unidades a partir de solo criterios conceptuales [...]. Las variables que delimitan la composición estructural de la muestra son definidas de manera teórica por el investigador” (p. 169). Por lo tanto, la agrupación de las categorías se elaboró con base en el conocimiento, criterio profesional y juicio del investigador. Específicamente, se eligieron individuos que tienen particularidades notables y características claras para la investigación, y se puso el foco en 57 proyectos de grado de las cohortes I, II, III y IV, de los cuales once se realizaron en pareja y dos fueron descartados porque no pudieron ser verificados para este estudio.

\section{Resultados}

La identificación de las tendencias de investigación es esencial para comprender la estructura temática de un dominio científico. El objetivo de este 
análisis fue identificar las principales lineas de investigación dentro del dominio de las ciencias militares aeronáuticas entre los años 2016-2020.

Para ello se seleccionaron los trabajos de grado de la MaCma alojados a 30 de junio de 2020 en el Catálogo Bibliográfico del Sistema de Bibliotecas de las Fuerzas Armadas (SIBFA). Las unidades de análisis utilizadas fueron las palabras clave, temas de investigación y objetos de investigación extraídas de forma automática del Social Science Citation Index (SscI) a través de la aplicación de Mendeley. Con las palabras clave y los temas de investigación que se obtuvieron se crearon mapas de etiquetado y de densidad, aplicando una metodología basada en el análisis de coocurrencia de palabras.

Como se mencionó antes, se analizaron 57 documentos, con una frecuencia de tres palabras, los cuales se elaboraron entre el 2016 y 2020, según los análisis de los mapas. Cabe señalar que los mapas mostraron de forma sintética (figura 2) la temática y permitieron visualizar la trayectoria investigativa en el campo de las CMA en la MACMA. En este sentido, dado el carácter multidisciplinar y transdisciplinar de las CMA, se puede afirmar que los mapas bidimensionales constituyen un instrumento adecuado para comprender la estructura temática de este campo.

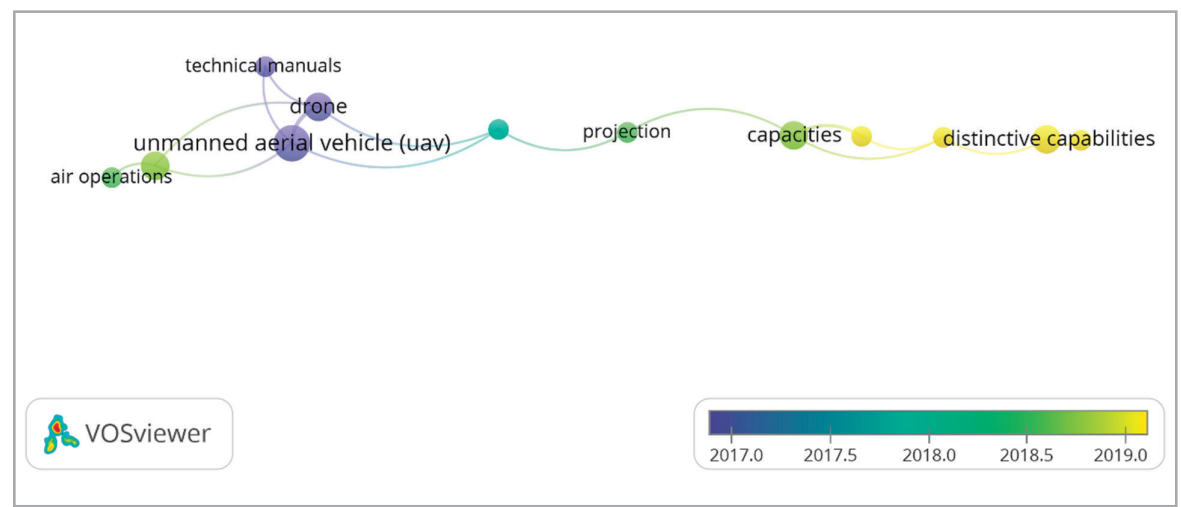

Figura 2. Mapa de etiquetado que muestra los temas de la trayectoria investigativa de las ciencias militares aeronáuticas en el periodo 2017-2019.

Fuente: Elaboración propia.

Las temáticas en la línea de tiempo 2017 a 2019 se puede observar que la mayor agrupación de temas de investigación está en el 2017, como lo muestra 
la figura 2, y han evolucionado hasta agruparse en cuatro núcleos de conocimiento, a saber:

- Vehículos aéreos no tripulados (UAV, por su sigla en inglés) (drones y manuales y técnicas).

- Proyección.

- $\quad$ Capacidades.

- $\quad$ Capacidades distintivas.

Por tanto, se infiere por los requerimientos de investigación de la MACMA que los trabajos están enfocados tanto en solucionar problemas de la FAC como en explorar capacidades y uso del poder aéreo, así como, específicamente, en generar doctrina (conocimiento) a través de la capacitación y estudio de las nuevas tecnologías, en este caso el desarrollo tecnológico de drones o UAV.

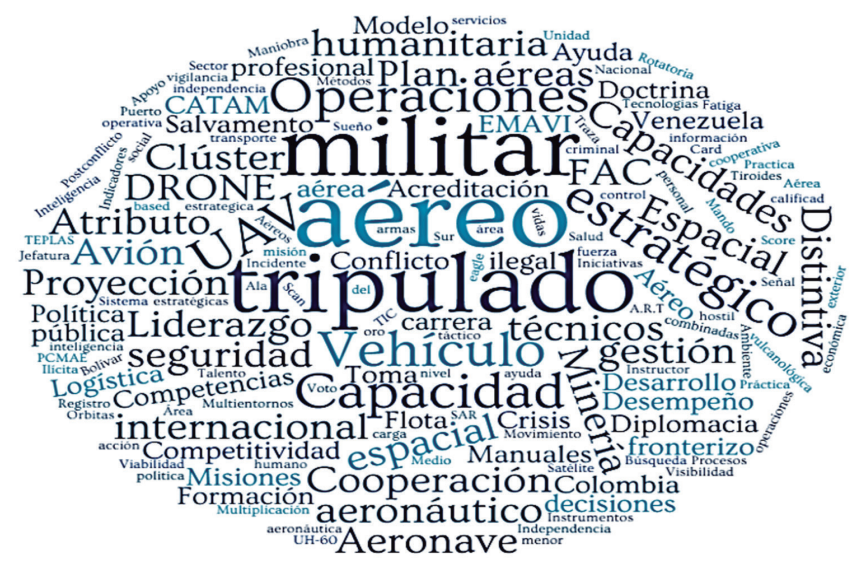

Figura 3. Nube de palabras de los temas investigados en los trabajos de grado 2016-2019 en la Macma.

Fuente: Elaboración propia usando el recurso Nube de Palabras (Zygomatic, s. f.).

En concordancia, la figura 3 muestra la asociación de palabras clave obtenidas del procesamiento de los datos de los trabajos de grado estudiados, esquematiza los objetos de investigación desarrollados desde el 2017 en los proyectos de grado de los estudiantes de la MaCMa y se complementa con los temas investigados por los estudiantes, como se visualiza en la figura 4. 


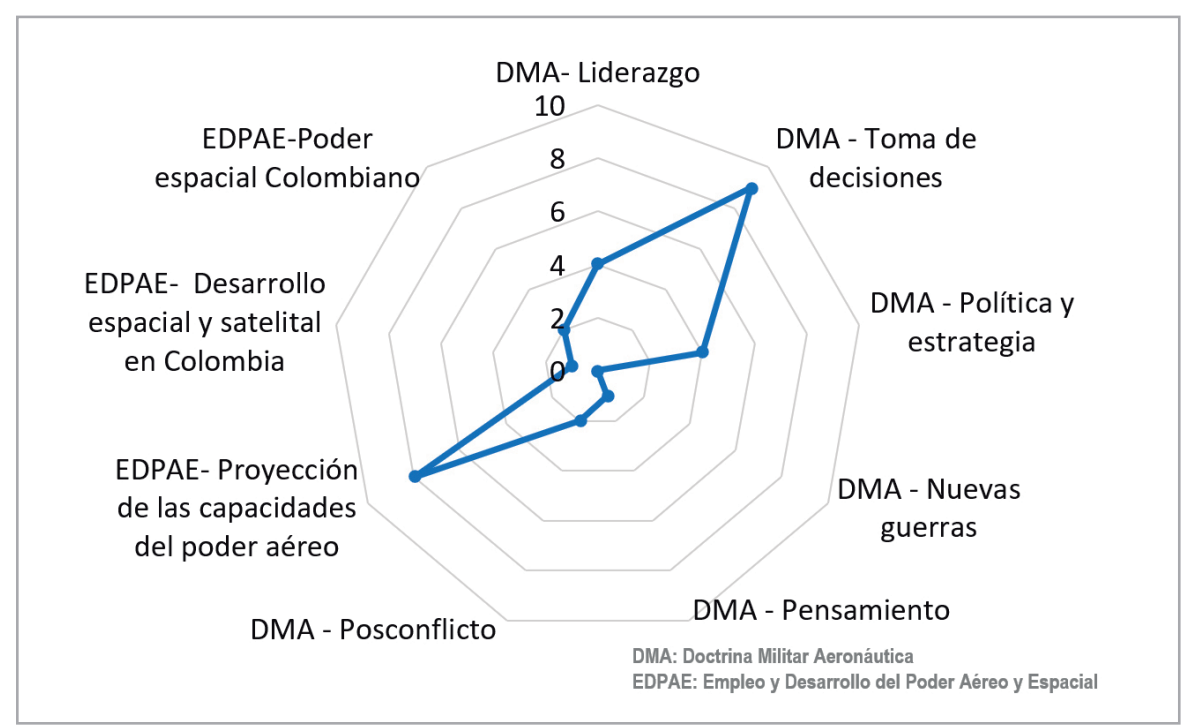

Figura 4. Temas investigados como proyectos de grado en la Macma.

Fuente: Elaboración propia.

\section{Discusión}

Este análisis ofrece una perspectiva diferente en relación con el estudio de las ciencias militares en Colombia. El componente aeronáutico y, recientemente, el espacial, así como el interés por el estudio y la comprensión de las nuevas tecnologías que fortalecen las capacidades del poder aéreo, su empleo y sostenimiento, le dan identidad académica a la MACMA al abordar, a través de los proyectos de grado de sus estudiantes, objetos de estudio que escapan al interés hasta ahora generalizado en el estudio de la doctrina y las ciencias militares, con lo cual amplía el campo de conocimiento de las CMA y promueve igualmente la producción de nuevo conocimiento, es decir, de nueva doctrina.

\section{Conclusiones}

La finalidad de la enseñanza universitaria es transmitir los conocimientos y desarrollar competencias de alto nivel en los egresados. Al analizar la trayectoria investigativa de las ciencias militares aeronáuticas (CMA) en Colombia, con el propósito de determinar los horizontes investigativos en la Maestría 
en Ciencias Militares Aeronáuticas (МАCMA), se hizo una revisión sistemática respecto a la educación superior, la doctrina y las competencias investigativas. Asimismo, se caracterizaron 57 proyectos de grado alojados en la Biblioteca Digital EPFAC, y se identificaron los núcleos de investigación desarrollados por los estudiantes y docentes de la MaCMA desde sus inicios en el 2016 hasta el 2020, lo cual permite trazar un horizonte de investigación en el campo de las CMA a mediano y largo plazo.

Según los hallazgos que evidencian los mapas de ocurrencia, los temas investigativos se ajustan a la malla curricular de la MACMA, y las investigaciones se han orientado al desarrollo tecnológico y el interés por explorar aspectos como las capacidades en UAV, por ejemplo (Acuña, 2016; Gomis-Balestreri \& Falck, 2015; Osorio et al., 2013).

En definitiva, de acuerdo con los trabajos de grado desarrollados en la MACMA se infiere que la trayectoria investigativa de las CMA ha tendido al estudio de los siguientes temas: UAV-drones, manuales y técnicas, proyección, capacidades y capacidades distintivas.

Por lo tanto, el desarrollo de investigaciones que contribuyan o permitan que la Fuerza Aérea esté a la vanguardia implica trabajar en la constante búsqueda y construcción de análisis coyunturales en escenarios de participación y acción, así como en nuevas interpretaciones ante la realidad contemporánea no solo de nuestro país, sino también del mundo.

En síntesis, la investigación en las CMA evoluciona, y por ello exige currículos flexibles e integradores (Kemmis, 1993, citado en Estrada et al., 2018) que respondan a la demanda del entorno. En ese sentido, la Macma no puede ser ajena a dinamizar su trayectoria investigativa para generar conocimientos y garantizar estudios actualizados y acordes a las necesidades de la institución. Así lo menciona Echavarría (2016) en relación con las intenciones y objetivos de los posgrados en profundización: "La educación superior está relacionada con la creación de conocimiento, estructura disciplinaria, práctica investigativa, contexto de aplicación, transdisciplinariedad, heterogeneidad y diversidad organizacional, responsabilidad social y control de calidad" (p. 266).

En suma, los resultados de este análisis le ofrecen a la MACMA, así como a las ofertas educativas similares, información de consulta que contribuye a la 
trayectoria investigativa que emerge del currículo, al tiempo que aportan al fortalecimiento de las competencias inherentes a la profesión y a la producción de conocimiento en el campo de las CMA. De esta manera, esta investigación hace una gran contribución al estudio de la doctrina y las ciencias militares en Colombia.

\section{Referencias}

Acuña, M. E. (2016). Drones, nuevos panoramas para la aviación: Análisis comparativo de la normatividad internacional frente a la normatividad colombiana. Ciencia y Poder Aéreo, 11(1). https://doi.org/10.18667/cienciaypoderaereo.524

Decreto 1330 de 2019. (2019). "Por el cual se sustituye el Capítulo 2 y se suprime el Capítulo 7 del Título 3 de la Parte 5 del Libro 2 del Decreto 1075 de 2015 - Único Reglamentario del Sector Educación”. Ministerio de Educación Nacional. https://www.mineducacion. gov.co/1759/articles-387348_archivo_pdf.pdf

Echavarría, C., Rodríguez, G., Bernal, J., \& González, L. (2016). La formación avanzada: Alcances y desafíos para el desarrollo social y político. Ciencia y Poder Aéreo, 11(1). https://doi.org/10.18667/cienciaypoderaereo.520

Escuela de Postgrados Fuerza Aérea Colombiana [EPFAC]. (2019). Documento maestro ciencias militares aeronáuticas.

Esquivel, T. (2015). Modernidad hispana en las ciencias militares en Colombia. Revista Científica General José María Córdova, 13(15), 291-307. https://doi:10.21830/19006586.28

Estrada Villa, E., Boude Figueredo, O. R., \& Martínez Lobo, A. P. (comp.). (2018). El M-Learning en la educación superior en Colombia: Un caso de aplicación. Fuerza Aérea Colombiana. https://doi.org/10.18667/9789585996151

Fuerza Aérea Colombiana [FAC]. (2018). Manual del Modelo de Investigación del Sistema Educativo de la Fuerza Aérea Colombiana (MAINV) FAC-7.20C. FAC.

Fuerza Aérea Colombiana [FAC]. (2020). Manual de Doctrina Básica Aérea y Espacial (5.a ed.). FAC.

Gomis-Balestreri, M., \& Falck, F. (2015). De ficción a realidad: Drones y seguridad ciudadana en América Latina. Ciencia y Poder Aéreo, 10(1), 71-84. https://doi.org/10.18667/cienciaypoderaereo. 430

Hernández-Sampieri, R., Fernández, C., \& Baptista, P. (2010). Metodología de la investigación (5. ${ }^{\mathrm{a}}$ ed.). McGraw Hill.

Ley 30 de 1992. (1992). "Por la cual se organiza el servicio público de la Educación Superior". Congreso de la República, Diario Oficial n. ${ }^{\circ}$ 40.700. http://www.secretariasenado.gov. co/senado/basedoc/ley_0030_1992.html

Mejía, J. (2000). El muestreo en la investigación cualitativa. Investigaciones Sociales, 4(5), 165-180. https://doi.org/10.15381/is.v4i5.6851 
Ministerio de Defensa Nacional. (2008). PESE. Plan Estratégico del Sistema Educativo de las Fuerzas Armadas 2007-2019. https://www.mindefensa.gov.co/irj/go/km/docs/ Mindefensa/Documentos/descargas/estrategia_planeacion/desa_capital/Pagina/PESE_ FINAL.pdf

Morin, E. (2001). Los siete saberes necesarios para la educación del futuro. Magisterio.

Organización de las Naciones Unidas para la Educación, la Ciencia y la Cultura [UNESCO]. (2013). Clasificación Internacional Normalizada de la Educación. CINE 2011. UNESCO. http://uis.unesco.org/sites/default/files/documents/isced-2011-sp.pdf

Organización para la Cooperación y el Desarrollo Económicos [OCDE] (2015). The measurement of scientific, technological and innovation activities. Manual Frascati 2015: Guidelines for collecting and reporting data on research and experimental development. OCDE. https://doi.org/10.1787/9789264239012-en

Ortega, R. (2016). Ciencias militares. Ciencia - arte - método - Estado Mayor. Departamento de Estudios Estratégicos-Ejército de Chile.

Osorio, A., Tamayo, A., Alvarado, J., Barragán, G., \& Urrea, G. (2013). Selección de materiales para la estructura de una aeronave de ala rotatoria no tripulada. Ciencia y Poder Aéreo, 8(1), 42-46. https://doi.org/10.18667/cienciaypoderaereo.6

Pérez Rocha, M. I. (2012). Fortalecimiento de las competencias investigativas en el contexto de la educación superior en Colombia. Revista de Investigaciones UNAD, 1(11), 9-34. https://doi.org/10.22490/25391887.770

Pimienta, J. (2012). Las competencias en la docencia universitaria. Pearson Educación. https://www.academia.edu/33825697/Las_competencias_en_la_docencia_universitaria_ pimienta_1_

Reiban Barrera, R. E, De la Rosa Rodríguez, H., \& Zeballos Chang, J. M. (2017). Competencias investigativas en la educación superior. Revista Publicando, 4(10-1), 395-405. https:// core.ac.uk/download/pdf/236644238.pdf

Suárez, P. (2005). ¿Qué son las ciencias militares? Revista Cientifica General José María Córdova, 3(3), 45-48. https://www.redalyc.org/pdf/4762/476259066012.pdf

Tobón, S. (2008). La formación basada en competencias en la educación superior: El enfoque complejo [Documento de trabajo, Curso IGLU 2008]. Universidad Autónoma de Guadalajara. CIFE. https://www.researchgate.net/publication/329440312_La_formacion_basada_en_ competencias_en_la_educacion_superior_el_enfoque_complejo

Zygomatic. (s. f.). Nube de Palabras [recurso en línea]. https://www.nubedepalabras.es/ 